\title{
Normal-ordering approximations and translational (non)invariance
}

\author{
T. Djärv $\odot,{ }^{1, *}$ A. Ekström, ${ }^{1}$ C. Forssén $\odot,{ }^{1, \dagger}$ and G. R. Jansen $\circledast^{2,3}$ \\ ${ }^{1}$ Department of Physics, Chalmers University of Technology, SE-412 96 Gothenburg, Sweden \\ ${ }^{2}$ National Center for Computational Sciences, Oak Ridge National Laboratory, Oak Ridge, Tennessee 37831, USA \\ ${ }^{3}$ Physics Division, Oak Ridge National Laboratory, Oak Ridge, Tennessee 37831, USA
}

(Received 7 May 2021; accepted 11 August 2021; published 23 August 2021)

\begin{abstract}
Normal ordering provides an approach to approximate three-body forces as effective two-body operators and it is therefore an important tool in many-body calculations with realistic nuclear interactions. The corresponding neglect of certain three-body terms in the normal-ordered Hamiltonian is known to influence translational invariance, although the magnitude of this effect has not yet been systematically quantified. In this paper we study in particular the normal-ordering two-body approximation applied to a single harmonic-oscillator reference state. We explicate the breaking of translational invariance and demonstrate the magnitude of the approximation error as a function of model space parameters for ${ }^{4} \mathrm{He}$ and ${ }^{16} \mathrm{O}$ by performing full no-core shell-model calculations with and without three-nucleon forces. We combine two different diagnostics to better monitor the breaking of translational invariance. While the center-of-mass effect is shown to become potentially very large for ${ }^{4} \mathrm{He}$, it is also shown to be much smaller for ${ }^{16} \mathrm{O}$ although full convergence is not reached. These tools can be easily implemented in studies using other many-body frameworks and bases.
\end{abstract}

DOI: 10.1103/PhysRevC.104.024324

\section{INTRODUCTION}

The need for an effective three-nucleon force (3NF) to describe the strong nuclear interaction in atomic nuclei is well established [1]. Its origin dates back to Fujita and Miyazawa, who computed the $3 \mathrm{NF}$ arising from a two-pion exchange diagram [2]. In the modern understanding, 3NFs arise in effective field theories (EFTs) as a consequence of integrating out degrees of freedom. More specifically, 3NFs appear in the chiral EFT of the strong nuclear interaction at next-to-next-toleading order (N2LO) and above in standard power counting schemes of the chiral expansion [3-6]. Besides the EFT arguments, it has also been shown that several experimental findings are difficult to reproduce without the inclusion of a $3 \mathrm{NF}$, such as certain three-nucleon scattering observables [7], the $A=3,4$ binding energies [8,9], and selected light nucleus spectroscopy [10-12].

Unfortunately, the full inclusion of $3 \mathrm{NFs}$ in quantum many-body methods is computationally demanding due to the large increase in the number of nonzero matrix elements [13]. In fact, the escalation of memory demands and the increase in execution time often render solutions intractable when explicit 3NFs are added. This situation has initiated searches for

\footnotetext{
*djarv@ chalmers.se

${ }^{\dagger}$ christian.forssen@chalmers.se
}

Published by the American Physical Society under the terms of the Creative Commons Attribution 4.0 International license. Further distribution of this work must maintain attribution to the author $(s)$ and the published article's title, journal citation, and DOI. Funded by Bibsam. approximation schemes that will include the most important physics of $3 \mathrm{NFs}$, but at a lower computational cost.

One such approximation scheme is the single-reference normal-ordering two-body (SR-NO2B) approximation $[14,15]$, which potentially can incorporate the dominant piece of the $3 \mathrm{NF}$ as an effective two-nucleon force $(2 \mathrm{NF})$ and therefore at significantly reduced computational cost. This is often done by approximating the ground state with a single Slater determinant (SD) and then normal ordering the $3 \mathrm{NF}$ relative to this reference state using Wick's theorem [16]. The expectation value of the residual three-nucleon term, acting only outside the reference state, is assumed to give a much smaller contribution to the ground-state energy than the induced two-, one-, and zero-body parts-and is therefore discarded.

The SR-NO2B approximation has been used with great success in $a b$ initio nuclear structure calculations-in particular to facilitate studies of medium-mass systems [15,17-20]. Normal-ordering approximations beyond the single reference state have also been developed [21,22]. The accuracy of the SR-NO2B has been benchmarked, e.g., in Refs. [14,15,23]. The importance of residual 3NFs was shown to be small by explicit comparison with calculations using full 3NFs. However, these benchmarks were performed at a fixed oscillator frequency and the dependence on model-space parameters has not been investigated. This is particularly important since we show that the sensitivity of SR-NO2B to the choice of basis frequency could be significant.

We stress that our concern pertains to the explicit breaking of translational invariance of the underlying Hamiltonian due to the normal-ordering two-body (NO2B) approximation. The ensuing center-of-mass (CM) dependence is therefore 
of different origin compared to the well-known problem of $\mathrm{CM}$ mixing as a consequence of, e.g., particular truncations of the single-particle basis that are used in some many-body solvers [20,24-29]. We also note that a conceptually different, but related, issue appears when working with symmetry broken (and restored) reference states. In that context, there have been studies to design more general normal-ordering approximations to obtain operators that are still consistent with symmetries of the Hamiltonian. In particular, such designs exist for reference states with broken particle-number symmetry [30], and rotational symmetry [31].

In this paper, we have studied the SR-NO2B approximation in a harmonic oscillator $(\mathrm{HO})$ basis with a no-core shell model (NCSM) total-energy truncation. We consider the closed-shell systems ${ }^{4} \mathrm{He}$ and ${ }^{16} \mathrm{O}$ - for which the single-reference approximation is appropriate-and we explore the accuracy of the $\mathrm{NO} 2 \mathrm{~B}$ approximation and the breaking of translational invariance as a function of model space parameters $N_{\max }$ and $\hbar \Omega$. The realistic $\mathrm{N} 2 \mathrm{LO}_{\text {sat }}$ interaction [32] with both $2 \mathrm{NF}$ and $3 \mathrm{NF}$ terms is used for all numerical calculations unless otherwise stated.

The full Hamiltonian, the NCSM method, and the SRNO2B approximation are introduced in Sec. II. The CM problem is presented in Sec. III where we also introduce and benchmark the metrics that will be used in the analysis. The NO2B approximation errors for ground-state energies and radii for ${ }^{4} \mathrm{He}$ and ${ }^{16} \mathrm{O}$ are analyzed in Sec. IV, while concluding remarks are given in Sec. V.

\section{THEORY}

\section{A. The Hamiltonian}

The general Hamiltonian that is considered in this paper can be written

$$
\hat{H}=\hat{T}_{\text {int }}+\hat{V}_{2 \mathrm{NF}}+\hat{V}_{3 \mathrm{NF}} .
$$

The potential operators are expressed in second quantized form as

$$
\hat{V}_{2 \mathrm{NF}}=\frac{1}{4} \sum_{\substack{\alpha \beta \\ \alpha^{\prime} \beta^{\prime}}}\left\langle\alpha \beta\left|V_{2 \mathrm{NF}}\right| \alpha^{\prime} \beta^{\prime}\right\rangle \hat{c}_{\alpha}^{\dagger} \hat{c}_{\beta}^{\dagger} \hat{c}_{\beta^{\prime}} \hat{c}_{\alpha^{\prime}},
$$

and

$$
\hat{V}_{3 \mathrm{NF}}=\frac{1}{36} \sum_{\substack{\alpha \beta \gamma \\ \alpha^{\prime} \beta^{\prime} \gamma^{\prime}}}\left\langle\alpha \beta \gamma\left|V_{3 \mathrm{NF}}\right| \alpha^{\prime} \beta^{\prime} \gamma^{\prime}\right\rangle \hat{c}_{\alpha}^{\dagger} \hat{c}_{\beta}^{\dagger} \hat{c}_{\gamma}^{\dagger} \hat{c}_{\gamma^{\prime}} \hat{c}_{\beta^{\prime}} \hat{c}_{\alpha^{\prime}}
$$

with Greek letters representing tuples of the well-known single-particle quantum numbers $\left(n, l, j, j_{z}, t_{z}\right)$ in a $\mathrm{HO}$ basis. These operators, as well as the intrinsic kinetic energy, $\hat{T}_{\text {int }}$, depend on relative coordinates (in position and momentum space) such that the Hamiltonian (1) is translationally invariant.

\section{B. The no-core shell model}

To solve the many-body Schrödinger equation we employ the NCSM in which the Schrödinger equation

$$
\hat{H}|\Psi\rangle=E|\Psi\rangle
$$

is rewritten as a finite matrix eigenvalue problem by expanding the eigenstates of the Hamiltonian $\hat{H}$ in a finite many-body basis $\left\{\left|\Phi_{i}\right\rangle\right\}_{i=1}^{D}$, i.e., the NCSM eigenstate $n$ is

$$
\left|\Psi_{n}\right\rangle_{\mathrm{NCSM}}=\sum_{i=0}^{D} c_{n, i}\left|\Phi_{i}\right\rangle .
$$

The SD many-body basis state $\left|\Phi_{i}\right\rangle$ is constructed using second-quantization

$$
\left|\Phi_{i}\right\rangle=\hat{c}_{\alpha_{i, 1}}^{\dagger} \cdots \hat{c}_{\alpha_{i, A}}^{\dagger}|\rangle
$$

and is an eigenstate of a two-component $A$-body $(A=N+Z)$ fermionic HO Hamiltonian with the corresponding eigenenergy $E_{i}=\left(N_{i}+\frac{3}{2}\right) \hbar \Omega$ where $\Omega$ is the oscillator frequency and $N_{i}$ is the total $\mathrm{HO}$ energy quantum number

$$
N_{i} \equiv \sum_{n_{j}, l_{j} \in \Phi_{i}}\left(2 n_{j}+l_{j}\right),
$$

where $n_{j}\left(l_{j}\right)$ is the principle quantum number (orbital angular momentum) of particle $j$ in the basis state $\Phi_{i}$. The dimension $D$ of the NCSM basis is set by a total HO-energy truncation

$$
N_{i}-N_{\text {ref }} \leqslant N_{\max }
$$

where $N_{\text {ref }}$ is the total HO energy quantum number of a reference state composed of the $(N, Z)$ lowest single-particle $\mathrm{HO}$ states. For example, $N_{\text {ref }}=0$ for ${ }^{4} \mathrm{He}$ and $N_{\text {ref }}=12$ for ${ }^{16} \mathrm{O}$.

In general, there is no guarantee that the separation of intrinsic and $\mathrm{CM}$ excitations due to the translational invariance of the Hamiltonian is preserved when the Hilbert space is arbitrarily truncated. However, an important feature of the total-energy truncation of the NCSM basis is that it does in fact guarantee this separation due to the energy-conserving property of the HO transformation brackets [11]. This property implies that there exists a unitary mapping of a SD basis of HO single-particle states-truncated with respect to the HO excitation energy $N_{\max } \hbar \Omega$ - onto a Jacobi-coordinate basis. Therefore, the NCSM eigenstates of (1) can formally be written as product states

$$
\left|\Psi_{n}\right\rangle_{\mathrm{NCSM}}=\left|\Psi_{i}\right\rangle_{\mathrm{int}} \otimes\left|\Psi_{j}\right\rangle_{\mathrm{CM}},
$$

with the state number $n=n(i, j)$. The lowest energy state $(n=0)$ will be the product of the ground state of the CM motion $(j=0)$ and that of the intrinsic Hamiltonian $(i=0)$.

\section{Single-reference normal ordering}

In this section we outline the major steps of singlereference normal ordering and describe the NO2B approximation. We start from the general expression of the vacuum normal-ordered $3 \mathrm{NFs}$ in Eq. (3) and a reference state, that is, a single $\mathrm{SD}$ :

$$
\left|\psi_{\text {ref }}\right\rangle=\hat{c}_{\alpha_{1}}^{\dagger} \cdots \hat{c}_{\alpha_{A}}^{\dagger}|\rangle,
$$


constructed from $\mathcal{R}=\mathcal{R}(A, Z)=\left\{\alpha_{i}\right\}_{i=1}^{A}$-the lowest HO states for the $A$-body system composed of $Z$ protons and $N=A-Z$ neutrons.

$\hat{V}_{3 \mathrm{NF}}$ can then be normal ordered relative to $\left|\psi_{\text {ref }}\right\rangle$, which results in an expansion of zero-, one-, two-, and three-body operators. The contribution to the ground-state energy of the residual three-nucleon operator is assumed to be small-since it acts solely outside the reference state-and is discarded. This is known as the NO2B approximation as it results in an effective Hamiltonian with at most two-body operators.

The normal ordering relative to $\left|\psi_{\text {ref }}\right\rangle$ is easiest performed with Wick's theorem [33]. A product of second-quantization operators, normal ordered relative to $\left|\psi_{\text {ref }}\right\rangle$, is here written as $\{\hat{a} \hat{b} \hat{c} \cdots\}$. Such a normal-ordered operator fulfills $\{\hat{a} \hat{b} \hat{c} \cdots\}\left|\psi_{\text {ref }}\right\rangle=0$. Combined with the formal definition of a contraction, $\hat{a} \hat{b}=\hat{a} \hat{b}-\{\hat{a} \hat{b}\}$, it is possible to derive the contraction rules

$$
\begin{aligned}
& \hat{c}_{\alpha}^{\dagger} \hat{c}_{\beta}=\left\{\begin{array}{lc}
\delta_{\alpha, \beta} & \text { if } \alpha \in \mathcal{R}(A, Z) \wedge \beta \in \mathcal{R}(A, Z) \\
0 & \text { otherwise }
\end{array},\right. \\
& \hat{c}_{\alpha} \hat{c}_{\beta}^{\dagger}=\left\{\begin{array}{lc}
\delta_{\alpha, \beta} & \text { if } \alpha \notin \mathcal{R}(A, Z) \wedge \beta \notin \mathcal{R}(A, Z) \\
0 & \text { otherwise }
\end{array}\right. \\
& \hat{c}_{\alpha}^{\dagger} \hat{c}_{\beta}^{\dagger}=0, \\
& \hat{c}_{\alpha} \hat{c}_{\beta}=0 \text {. }
\end{aligned}
$$

The $3 \mathrm{NF}$ in Eq. (3) can now be normal ordered relative to $\left|\psi_{\text {ref }}\right\rangle$ by applying Wick's theorem:

$$
\begin{aligned}
& \hat{V}_{3 \mathrm{NF}}=\overbrace{\frac{1}{6} \sum_{\alpha, \beta, \gamma \in \mathcal{R}}\left\langle\alpha \beta \gamma\left|V_{3 \mathrm{NF}}\right| \alpha \beta \gamma\right\rangle}^{\equiv W_{0}} \\
& +\overbrace{\frac{1}{2} \sum_{\substack{\alpha \\
\alpha^{\prime}}} \sum_{\beta, \gamma \in \mathcal{R}}\left\langle\alpha \beta \gamma\left|V_{3 \mathrm{NF}}\right| \alpha^{\prime} \beta \gamma\right\rangle\left\{\hat{c}_{\alpha}^{\dagger} \hat{c}_{\alpha^{\prime}}\right\}}^{\equiv \hat{W}_{1}} \\
& +\overbrace{\frac{1}{4} \sum_{\substack{\alpha \beta \\
\alpha^{\prime} \beta^{\prime}}} \sum_{\gamma \in \mathcal{R}}\left\langle\alpha \beta \gamma\left|V_{3 \mathrm{NF}}\right| \alpha^{\prime} \beta^{\prime} \gamma\right\rangle\left\{\hat{c}_{\alpha}^{\dagger} \hat{c}_{\beta}^{\dagger} \hat{c}_{\beta^{\prime}} \hat{c}_{\alpha^{\prime}}\right\}}^{\equiv \hat{W}_{2}} \\
& +\overbrace{\frac{1}{36} \sum_{\substack{\alpha \beta \gamma \\
\alpha^{\prime} \beta^{\prime} \gamma^{\prime}}}\left\langle\alpha \beta \gamma\left|V_{3 \mathrm{NF}}\right| \alpha^{\prime} \beta^{\prime} \gamma^{\prime}\right\rangle\left\{\hat{c}_{\alpha}^{\dagger} \hat{c}_{\beta}^{\dagger} \hat{c}_{\gamma}^{\dagger} \hat{c}_{\gamma^{\prime}} \hat{c}_{\beta^{\prime}} \hat{c}_{\alpha^{\prime}}\right\}}^{\equiv \hat{W}_{3}},
\end{aligned}
$$

where we note that $W_{0}$ is a constant while $\hat{W}_{i}\left|\psi_{\text {ref }}\right\rangle=0$ for $i \in\{1,2,3\}$ due to the normal-ordered second-quantization operators. The NO2B approximation of $\hat{V}_{3 \mathrm{NF}}$ is then defined as

$$
\hat{V}_{3 \mathrm{NF}}^{\mathrm{NO} 2 \mathrm{~B}} \equiv W_{0}+\hat{W}_{1}+\hat{W}_{2}
$$

In the NCSM, however, the Hamiltonian is not expressed relative to a reference state and we need to apply Wick's theorem backwards to transform $\hat{V}_{3 \mathrm{NF}}^{\mathrm{NO} 2 \mathrm{~B}}$ into vacuum normal-ordered form. With this aim, we use the relations

$$
\begin{aligned}
\left\{\hat{c}_{\alpha}^{\dagger} \hat{c}_{\alpha^{\prime}}\right\}= & \hat{c}_{\alpha}^{\dagger} \hat{c}_{\alpha^{\prime}}-\hat{c}_{\alpha}^{\dagger} \hat{c}_{\alpha^{\prime}}, \\
\left\{\hat{c}_{\alpha}^{\dagger} \hat{c}_{\beta}^{\dagger} \hat{c}_{\beta^{\prime}} \hat{c}_{\alpha^{\prime}}\right\}= & \hat{c}_{\alpha}^{\dagger} \hat{c}_{\beta}^{\dagger} \hat{c}_{\beta^{\prime}} \hat{c}_{\alpha^{\prime}} \\
& -\hat{c}_{\beta}^{\dagger} \hat{c}_{\beta^{\prime}}\left\{\hat{c}_{\alpha}^{\dagger} \hat{c}_{\alpha^{\prime}}\right\}-\hat{c}_{\alpha}^{\dagger} \hat{c}_{\alpha^{\prime}}\left\{\hat{c}_{\beta}^{\dagger} \hat{c}_{\beta^{\prime}}\right\} \\
& +\hat{c}_{\beta}^{\dagger} \hat{c}_{\alpha^{\prime}}\left\{\hat{c}_{\alpha}^{\dagger} \hat{c}_{\beta^{\prime}}\right\}+\hat{c}_{\alpha}^{\dagger} \hat{c}_{\beta^{\prime}}\left\{\hat{c}_{\beta}^{\dagger} \hat{c}_{\alpha^{\prime}}\right\} \\
& -\hat{c}_{\alpha}^{\dagger} \hat{c}_{\alpha^{\prime}} \hat{c}_{\beta}^{\dagger} \hat{c}_{\beta^{\prime}}+\hat{c}_{\alpha^{\dagger}}^{\dagger} \hat{c}_{\beta^{\prime}} \hat{c}_{\beta}^{\dagger} \hat{c}_{\alpha^{\prime}}
\end{aligned}
$$

and arrive at

$$
\begin{aligned}
\hat{V}_{3 \mathrm{NF}}^{\mathrm{NO} 2 \mathrm{~B}}= & \frac{1}{6} \sum_{\alpha, \beta, \gamma \in \mathcal{R}}\left\langle\alpha \beta \gamma\left|V_{3 \mathrm{NF}}\right| \alpha \beta \gamma\right\rangle \\
& -\frac{1}{2} \sum_{\substack{\alpha \\
\alpha^{\prime}}} \sum_{\beta, \gamma \in \mathcal{R}}\left\langle\alpha \beta \gamma\left|V_{3 \mathrm{NF}}\right| \alpha^{\prime} \beta \gamma\right\rangle \hat{c}_{\alpha^{\dagger}}^{\dagger} \hat{c}_{\alpha^{\prime}} \\
& +\frac{1}{4} \sum_{\substack{\alpha \beta \\
\alpha^{\prime} \beta^{\prime}}} \sum_{\gamma \in \mathcal{R}}\left\langle\alpha \beta \gamma\left|V_{3 \mathrm{NF}}\right| \alpha^{\prime} \beta^{\prime} \gamma\right\rangle \hat{c}_{\alpha}^{\dagger} \hat{c}_{\beta}^{\dagger} \hat{c}_{\beta^{\prime}} \hat{c}_{\alpha^{\prime}} .
\end{aligned}
$$

In the end, the NO2B-approximated Hamiltonian that we use in the NCSM is

$$
\hat{H}^{\mathrm{NO} 2 \mathrm{~B}}=\hat{T}_{\mathrm{int}}+\hat{V}_{2 \mathrm{NF}}+\hat{V}_{3 \mathrm{NF}}^{\mathrm{NO} 2 \mathrm{~B}} .
$$

This is clearly different compared to the full Hamiltonian, $\hat{H}$, given by Eq. (1) where the complete $3 \mathrm{NF}$ is retained.

\section{THE CENTER-OF-MASS PROBLEM}

The translational symmetry of the NO2B-approximated Hamiltonian (20) is explicitly broken since we neglect the residual $3 \mathrm{NF}$ and this renders the $\mathrm{CM}$ dependence of the reference state manifest. Indeed, retaining the residual $3 \mathrm{NF}$ restores translational symmetry since the normal ordering in Eq. (15) is an exact relation. With the NO2B approximation it is therefore no longer guaranteed that the ground state $\left|\Psi_{\mathrm{GS}}^{\mathrm{NO} 2 \mathrm{~B}}\right\rangle_{\mathrm{NCSM}}$ of $\hat{H}^{\mathrm{NO} 2 \mathrm{~B}}$ is factorized into a product of CM and intrinsic states as in Eq. (9). Instead we must expect a linear superposition of product states:

$$
\left|\Psi_{\mathrm{GS}}^{\mathrm{NO} 2 \mathrm{~B}}\right\rangle_{\mathrm{NCSM}}=\sum_{i, j} c_{i, j}\left|\Psi_{i}\right\rangle_{\mathrm{int}} \otimes\left|\Psi_{j}\right\rangle_{\mathrm{CM}} .
$$

In this more general situation the intrinsic and CM states are no longer pure quantum states and must be expressed with density matrices $\hat{\rho}_{\text {int }}$ and $\hat{\rho}_{\mathrm{CM}}$.

This mixing of CM and intrinsic degrees of freedom can potentially have a huge effect on various observables and is here labeled as the center-of-mass problem. It is therefore crucial to quantify the CM mixing. In the following we will introduce two metrics that have exactly this purpose. 


\section{A. Introducing center-of-mass metrics}

The mixing of CM and intrinsic states is a known problem in many-body physics. It might occur also when using fully translational-invariant Hamiltonians as a consequence of approximations used in the many-body solver. In particular, Galilean invariance is broken explicitly when employing lattice methods [34] and CM mixing can occur in basisexpansion methods when imposing a basis truncation at the single-particle level [20,24-29]. A very common approach to diagnose the problem in basis expansion methods is to evaluate the (energy-shifted) HO CM Hamiltonian

$$
\hat{H}_{\mathrm{CM}}(\omega)=\frac{\hat{P}_{\mathrm{CM}}^{2}}{2 m A}+\frac{1}{2} m A \omega^{2} \hat{R}_{\mathrm{CM}}^{2}-\frac{3}{2} \hbar \omega,
$$

or the corresponding $\mathrm{CM}$ number operator

$$
\hat{N}_{\mathrm{CM}}(\omega)=\frac{1}{\hbar \omega} \hat{H}_{\mathrm{CM}}(\omega)
$$

with expectation value $N_{\mathrm{CM}}(\omega)$. Small expectation values of these operators, $\hat{H}_{\mathrm{CM}}(\Omega)$ and $\hat{N}_{\mathrm{CM}}(\Omega)$, evaluated at the basis frequency $\Omega$, are then used as evidence for satisfactory CM factorization. Large expectation values, on the other hand, indicate problematic mixing.

However, it might be too assertive to claim proper CM separation based on this single observable. We argue here that additional metrics are needed. In addition, there are claims [25] that the factorization does occur but that the CM state is not necessarily a ground state of the Hamiltonian $\hat{H}_{\mathrm{CM}}(\Omega)$ (22) constructed using the basis frequency $\hbar \Omega$.

\section{The $\xi_{C M}$ metric}

Consider an eigenstate of a translationally invariant Hamiltonian that factorizes into a product of an intrinsic state $|\Psi\rangle_{\text {int }}$ and a CM state $\left|\Psi_{\mathrm{GS}}^{\omega_{\xi}}\right\rangle_{\mathrm{CM}}$, where the latter corresponds to the ground state of $\hat{H}_{\mathrm{CM}}\left(\omega_{\xi}\right)$ - the Hamiltonian (22) constructed with an oscillator frequency $\omega_{\xi}$ which does not necessarily correspond to the basis frequency $\Omega$. In this situation we would obtain the expectation values

$$
\begin{aligned}
\left\langle R_{\mathrm{CM}}^{2}\right\rangle & =\frac{3}{2} b^{2} \\
\text { and }\left\langle P_{\mathrm{CM}}^{2}\right\rangle & =\frac{3}{2} \frac{\hbar^{2}}{b^{2}},
\end{aligned}
$$

with the oscillator length $b=b\left(\omega_{\xi}\right)=\sqrt{\hbar / A m \omega_{\xi}}$, and the expectation values are with respect to the full ground state. This fact was utilized by Parzuchowski et al. [27] in their study of transition operators within the in-medium similarity renormalization group framework. They introduced the quantity

$$
\xi_{\mathrm{CM}} \equiv \frac{\sqrt{\left\langle\hat{R}_{\mathrm{CM}}^{2}\right\rangle\left\langle\hat{P}_{\mathrm{CM}}^{2}\right\rangle}}{\hbar}-\frac{3}{2},
$$

which will evaluate to $\xi_{\mathrm{CM}}=0$ if $\left|\Psi_{\mathrm{GS}}^{\omega_{\xi}}\right\rangle_{\mathrm{CM}}$ is a $\mathrm{HO}$ ground state, regardless of the frequency $\omega_{\xi}$, while $\xi_{\mathrm{CM}}>0$ if it is not. Note, however, that a HO eigenstate with one frequency, $\omega$, cannot be exactly represented in a truncated HO basis with

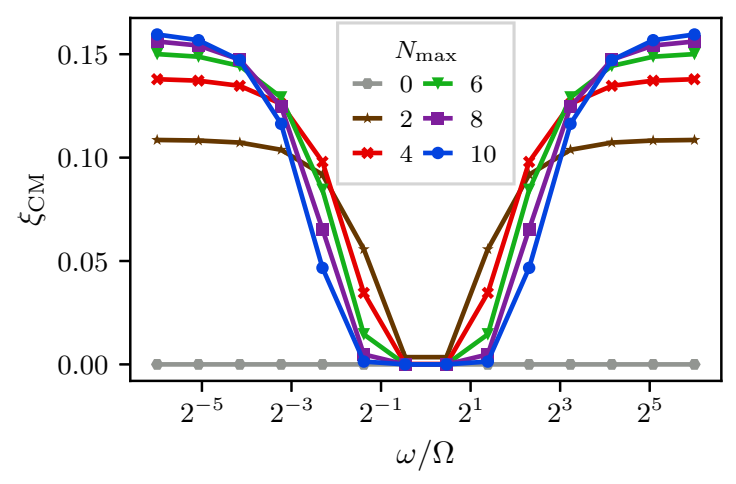

FIG. 1. The $\xi_{\mathrm{CM}}$ metric for ground states of $H_{\mathrm{CM}}(\omega)$ computed for different $\omega / \Omega$ ratios (where $\Omega$ is the basis frequency) and increasing NCSM basis truncations. We have pointwise convergence, $\xi_{\mathrm{CM}} \rightarrow 0$, for all $\omega / \Omega$ ratios as $N_{\max } \rightarrow \infty$.

a different basis frequency $\Omega \neq \omega$. This is illustrated in Fig. 1 and further discussed in the Appendix.

In the case when $\xi_{\mathrm{CM}} \approx 0$ it is possible to identify the corresponding frequency of the underlying HO Hamiltonian $\hat{H}_{\mathrm{CM}}\left(\omega_{\xi}\right)$ by

$$
\hbar \omega_{\xi}=\frac{4}{3}\left\langle\hat{T}_{\mathrm{CM}}\right\rangle
$$

where $\hat{T}_{\mathrm{CM}}$ is the CM kinetic energy, and the expectation value is with respect to the ground state. When the NCSM Hamiltonian is translationally invariant the frequency $\omega_{\xi}$ will equal the basis frequency in the NCSM method. However, $\omega_{\xi} \neq \Omega$ indicates a broken symmetry. Note that we might still have a product state (9) in this situation-such that CM mixing is not problematic_-and that we can measure this with $\xi_{\mathrm{CM}}$.

\section{The $N_{C M}$ metric}

In applications of the coupled-cluster (CC) method the computed ground state $\left|\Psi_{\mathrm{GS}}\right\rangle_{\mathrm{CC}}$ is assumed to be separable such that the CM state is the ground state of a HO Hamiltonian with a frequency $\omega_{N}$ that not necessarily equals the underlying HO basis frequency $\Omega$. The frequency $\omega_{N}$ is obtained by evaluating [25]

$$
\begin{aligned}
\hbar \omega^{ \pm}= & \hbar \Omega+\frac{2}{3}\left\langle\hat{H}_{\mathrm{CM}}(\Omega)\right\rangle \\
& \pm \sqrt{\frac{4}{9}\left\langle\hat{H}_{\mathrm{CM}}(\Omega)\right\rangle^{2}+\frac{4}{3} \hbar \Omega\left\langle\hat{H}_{\mathrm{CM}}(\Omega)\right\rangle},
\end{aligned}
$$

and identifying

$$
\omega_{N}=\arg \min { }_{\omega \in \omega^{ \pm}}\left\langle\hat{H}_{\mathrm{CM}}(\omega)\right\rangle .
$$

We can then define the operator $\hat{N}_{\mathrm{CM}}\left(\omega_{N}\right)$ analogous to Eq. (23) and evaluate its expectation value $N_{\mathrm{CM}}\left(\omega_{N}\right)$ which will be small if the wave function factorizes.

\section{B. The relation between different metrics}

There is an interesting connection between $\xi_{\mathrm{CM}}$ and $N_{\mathrm{CM}}\left(\omega_{\xi}\right)$, i.e., the two different metrics expressed in terms of the same CM oscillator frequency $\omega_{\xi}$. We pick $\omega_{\xi}$ since the 
two frequencies $\omega_{\xi}$ and $\omega_{N}$ are equal in the limit $N_{\max } \rightarrow \infty$ if the state is separable as in Eq. (9). Consider a factorized state with

$$
|\Psi\rangle_{\mathrm{CM}}=\left|\phi_{\mathcal{N} \mathcal{L}}^{\omega_{\xi}}\right\rangle
$$

i.e., it is a $\mathrm{HO}$ state with frequency $\omega_{\xi}$, radial quantum number $\mathcal{N}$, and orbital angular momentum $\mathcal{L}$. Then we have

$$
\begin{aligned}
N_{\mathrm{CM}}\left(\omega_{\xi}\right) & =2 \mathcal{N}+\mathcal{L}, \\
\left\langle R_{\mathrm{CM}}^{2}\right\rangle & =b\left(\omega_{\xi}\right)^{2}\left(2 \mathcal{N}+\mathcal{L}+\frac{3}{2}\right), \\
\text { and }\left\langle P_{\mathrm{CM}}^{2}\right\rangle & =\frac{\hbar^{2}}{b\left(\omega_{\xi}\right)^{2}}\left(2 \mathcal{N}+\mathcal{L}+\frac{3}{2}\right) .
\end{aligned}
$$

Using the definitions of the two metrics we find that they become equal in this scenario:

$$
\xi_{\mathrm{CM}}=\frac{\sqrt{\left\langle\hat{R}_{\mathrm{CM}}^{2}\right\rangle\left\langle\hat{P}_{\mathrm{CM}}^{2}\right\rangle}}{\hbar}-\frac{3}{2}=2 \mathcal{N}+\mathcal{L}=N_{\mathrm{CM}}\left(\omega_{\xi}\right) .
$$

Now consider the possibility that $|\Psi\rangle_{\mathrm{CM}}$ is a linear superposition

$$
|\Psi\rangle_{\mathrm{CM}}=\sum_{i} c_{i}\left|\phi_{\mathcal{N}_{i} \mathcal{L}_{i}}\right\rangle
$$

of HO states $\left|\phi_{\mathcal{N}_{i} \mathcal{L}_{i}}\right\rangle=a_{\mathcal{N}_{i} \mathcal{L}_{i}}^{\dagger}|\rangle$. To simplify further calculations we introduce

$$
\begin{aligned}
A= & \sum_{i}\left|c_{i}\right|^{2}\left(2 \mathcal{N}_{i}+\mathcal{L}_{i}+\frac{3}{2}\right) \\
\text { and } B= & \sum_{i, j} c_{i}^{*} c_{j}\left(\sqrt{\mathcal{N}_{i}\left(\mathcal{N}_{i}+\mathcal{L}_{i}+1 / 2\right)} \delta_{\mathcal{N}_{i}, \mathcal{N}_{j}+1}\right. \\
& \left.+\sqrt{\mathcal{N}_{j}\left(\mathcal{N}_{j}+\mathcal{L}_{i}+1 / 2\right)} \delta_{\mathcal{N}_{i}+1, \mathcal{N}_{j}}\right) \delta_{\mathcal{L}_{i}, \mathcal{L}_{j}}
\end{aligned}
$$

where $A, B$ are real and $A \geqslant 3 / 2$. Then we find

$$
\begin{aligned}
N_{\mathrm{CM}}\left(\omega_{\xi}\right) & =A-\frac{3}{2}, \\
\left\langle R_{\mathrm{CM}}^{2}\right\rangle & =b^{2}(A-B), \\
\text { and }\left\langle P_{\mathrm{CM}}^{2}\right\rangle & =\frac{\hbar^{2}}{b^{2}}(A+B),
\end{aligned}
$$

which gives

$$
\xi_{\mathrm{CM}}=\sqrt{A^{2}-B^{2}}-\frac{3}{2} .
$$

It is clear from Eqs. (38) and (41) that

$$
N_{\mathrm{CM}}\left(\omega_{\xi}\right)-\xi_{\mathrm{CM}}=\frac{A}{2}\left[\varepsilon+\mathcal{O}\left(\varepsilon^{2}\right)\right]
$$

where we have assumed that $\varepsilon \equiv B^{2} / A^{2} \ll 1$. Therefore, the difference between these two metrics can be used as a measure of how much of the CM state is in higher excitations. The off-diagonal sum $B$ can only be nonzero if there exists $i, j$ such that $c_{i}, c_{j} \neq 0$ with $\left|\mathcal{N}_{i}-\mathcal{N}_{j}\right|=1$ and $\mathcal{L}_{i}=\mathcal{L}_{j}$.

It turns out that a similar relation can be derived if we have $\mathrm{CM}$ mixing such that the CM state is not a pure quantum state. Then we find that $\xi_{\mathrm{CM}} \neq N_{\mathrm{CM}}$ if there does not exist any $\mathrm{HO}$ basis in which the CM density matrix

$$
\left(\rho_{\mathrm{CM}}\right)_{i, j}=\left\langle\Psi_{\mathrm{NCSM}}\left|a_{\mathcal{N}_{i} \mathcal{L}_{i}}^{\dagger} a_{\mathcal{N}_{j} \mathcal{L}_{j}}\right| \Psi_{\mathrm{NCSM}}\right\rangle
$$

is diagonal. In this situation the coefficients $A$ and $B$ in Eqs. (38) and (41) are given by

$$
\begin{aligned}
A= & \sum_{i}\left(\rho_{\mathrm{CM}}\right)_{i, i}\left(2 \mathcal{N}_{i}+\mathcal{L}_{i}+\frac{3}{2}\right) \\
\text { and } B= & \sum_{i, j}\left(\rho_{\mathrm{CM}}\right)_{i, j}\left(\sqrt{\mathcal{N}_{i}\left(\mathcal{N}_{i}+\mathcal{L}_{i}+1 / 2\right)} \delta_{\mathcal{N}_{i}, \mathcal{N}_{j}+1}\right. \\
& \left.+\sqrt{\mathcal{N}_{j}\left(\mathcal{N}_{j}+\mathcal{L}_{i}+1 / 2\right)} \delta_{\mathcal{N}_{i}+1, \mathcal{N}_{j}}\right) \delta_{\mathcal{L}_{i}, \mathcal{L}_{j}}
\end{aligned}
$$

In conclusion, when finding that $\xi_{\mathrm{CM}}, N_{\mathrm{CM}}>0$ we cannot know if the CM state is a pure quantum state or a mixed one. Only the situation $\xi_{\mathrm{CM}}=N_{\mathrm{CM}}=0$ assures a proper separation of the intrinsic and CM parts of the eigenstate as in Eq. (9).

\section{Benchmark of center-of-mass metrics}

To benchmark the CM analysis metrics we consider an interacting many-body system in an external HO trap with Hamiltonian

$$
\hat{H}^{\text {trap }}=\hat{T}_{\text {int }}+\hat{V}_{2 \mathrm{NF}}+\hat{H}_{\mathrm{CM}}\left(\omega_{\text {trap }}\right),
$$

where we use the $2 \mathrm{NF}$ part of $\mathrm{N} 2 \mathrm{LO}_{\text {sat }}$ as a realistic interaction $\hat{V}_{2 \mathrm{NF}}$. Then we compute the NCSM ground state of ${ }^{4} \mathrm{He}$ for different basis frequencies $\hbar \Omega \in\{8,12, \ldots, 32,36\} \mathrm{MeV}$ while keeping the trapping potential frequency fixed at $\hbar \omega_{\text {trap }}=$ $20 \mathrm{MeV}$. The metrics described in Sec. III A are then evaluated for the ground state.

While this Hamiltonian depends on the CM coordinatesuch that translational invariance is explicitly broken-it is still block diagonal in a CM part and an intrinsic part. Therefore, it is possible to precisely control the CM part of the ground state and this property makes it a suitable benchmark of the CM metrics. However, it is not equivalent to the non-block-diagonal CM coupling of the NO2B-approximated Hamiltonian.

We compute the expectation values $\left\langle R_{\mathrm{CM}}^{2}\right\rangle,\left\langle P_{\mathrm{CM}}^{2}\right\rangle$, and $\left\langle\hat{H}_{\mathrm{CM}}(\omega)\right\rangle$ for each NCSM model space $\left(N_{\max }, \hbar \Omega\right)$. This allows us to extract the optimal decoupling frequencies $\omega_{\xi}$ and $\omega_{N}$ and to test the decoupling by evaluating the metrics $\xi_{\mathrm{CM}}$ and $N_{\mathrm{CM}}\left(\omega_{N}\right)$. In addition, the standard CM-decoupling metric $N_{\mathrm{CM}}(\Omega)$ can be evaluated-although it is expected to fail when $\omega_{\text {trap }} \neq \Omega$. All of these quantities are plotted in Fig. 2 .

We numerically confirm that all three metrics, shown in the top row of Fig. 2, become equal to zero when the basis frequency $\Omega$ is equal to the trap frequency. However, while $N_{\mathrm{CM}}(\Omega)$ in panel (c) fails to reveal the decoupling for other basis frequencies, the two metrics $N_{\mathrm{CM}}\left(\omega_{N}\right)$ and $\xi_{\mathrm{CM}}$, shown in panels (a) and (b), respectively, do indicate decoupling by exhibiting small values.

The fact that both $N_{\mathrm{CM}}\left(\omega_{N}\right)$ and $\xi_{\mathrm{CM}}$ are larger for small basis frequencies indicates that a superposition of excited $\mathrm{HO}$ states is needed to describe the CM ground state in this truncated space (see the Appendix). However, for $\hbar \Omega>\hbar \omega_{\text {trap }}$ the metrics are very small already at modest $N_{\max }$, indicating a good CM state representation with these basis frequencies. 

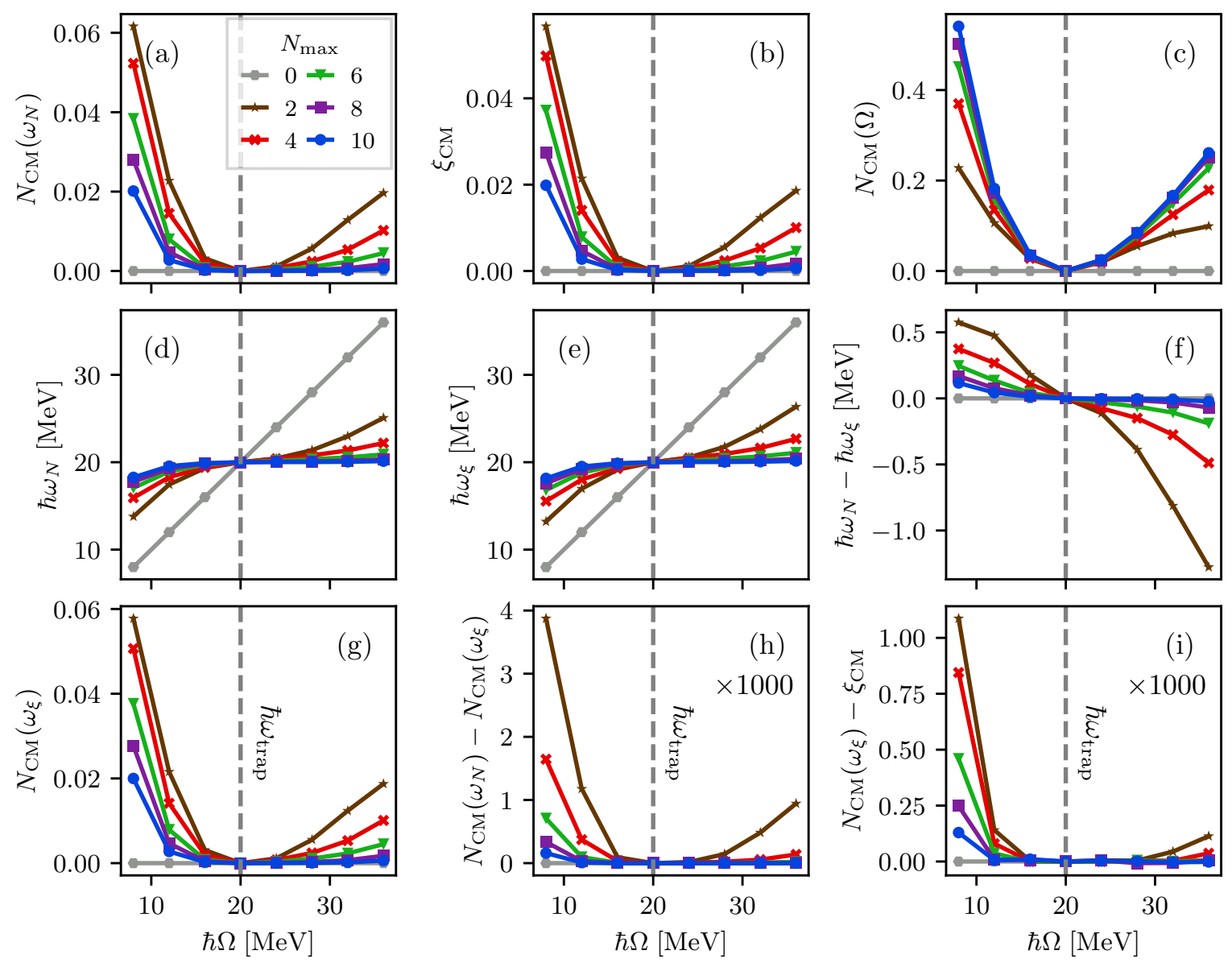

FIG. 2. CM-excitation metrics and HO frequencies of ${ }^{4} \mathrm{He}$ computed with an external trap $\hat{H}^{\text {trap }}$ (46) with $\hbar \omega_{\text {trap }}=20 \mathrm{MeV}$ using different basis frequencies $\hbar \Omega$. Note in particular that the two metrics $N_{\mathrm{CM}}$ and $\xi_{\mathrm{CM}}$ - shown in panels (a) and (b), respectively-are very similar and that both of them correctly identify the trap frequency for a wide range of basis frequencies-see panels (d) and (e). In contrast, the standard metric $N_{\mathrm{CM}}(\Omega)$, shown in panel (c), does not reveal the actual decoupling except for $\Omega=\omega_{\text {trap }}$. The differences shown in panels (h) and (i) are multiplied by a factor 1000 .

The corresponding optimal frequencies, $\hbar \omega_{N}$ and $\hbar \omega_{\xi}$ shown in panels (d) and (e), do approach the trap frequency as $N_{\max }$ increases. For $N_{\max }=0$, where there is a single SD basis state, this analysis will always return the basis frequency as the optimal one, as shown by the diagonal, straight line.

Finally, the differences between the optimal frequencies found via the $\xi_{\mathrm{CM}}$ and $N_{\mathrm{CM}}$ methods are shown in panel (f), while the differences between the two metrics are displayed in panels (h) and (i). As a general conclusion we find that the two analysis methods provide basically identical results, but that the $\xi_{\mathrm{CM}}$ metric is easier to implement and compute. Furthermore, the problem of representing a $\mathrm{HO}$ state of another frequency than that of the truncated $\mathrm{HO}$ basis hampers the analysis at small basis frequencies (see the Appendix).

\section{NO2B RESULTS}

In this section we present a numerical study of the SRNO2B approximation in the HO SD basis applied to the doubly magic systems ${ }^{4} \mathrm{He}$ and ${ }^{16} \mathrm{O}$. All results shown here are obtained with the realistic nuclear interaction model $\mathrm{N}_{2} \mathrm{LO}_{\text {sat }}[32]$. Throughout this paper we will compare results obtained with full and with NO2B-approximated 3NFs.
We employ the Jacobi-coordinate version of the NCSM $[35,36]$ to compute the ground state of ${ }^{4} \mathrm{He}$ with full $3 \mathrm{NF}$. The normal ordering is performed in the $M$-scheme SD basis and we employ the NCSM code PANTOINE $[37,38]$ to perform the diagonalization. Unfortunately, the huge number of $3 \mathrm{NF}$ matrix elements in the $M$-scheme SD basis limits our studies to model spaces $N_{\max } \leqslant 10$. Specifically, with $N_{\max }=10$ we have $5.4 \times 10^{9}$ elements while $N_{\max }=12$ would require $66.5 \times 10^{9}$.

For ${ }^{16} \mathrm{O}$ we are limited by the size and the number of nonzero elements of the Hamiltonian matrix. With NO2Bapproximated interactions we use PANTOINE and reach model spaces $N_{\max } \leqslant 8$ with up to $D=6 \times 10^{8}$ basis states. With full $3 \mathrm{NFs}$ we use the no-core shell-model Slater determinant (NCSD) code [39] and are able to reach model spaces $N_{\max } \leqslant 6$ corresponding to $D=1.6 \times 10^{6}$.

The direct comparison between results obtained with full and NO2B-truncated 3NFs allows us to focus on the size of the approximation error as a function of the mass number and model space parameters. The origin of the approximation error will here be analyzed in terms of possible CM mixing. In this context it is important to point out that all calculations in the $M$-scheme SD basis are performed without a Lawson 


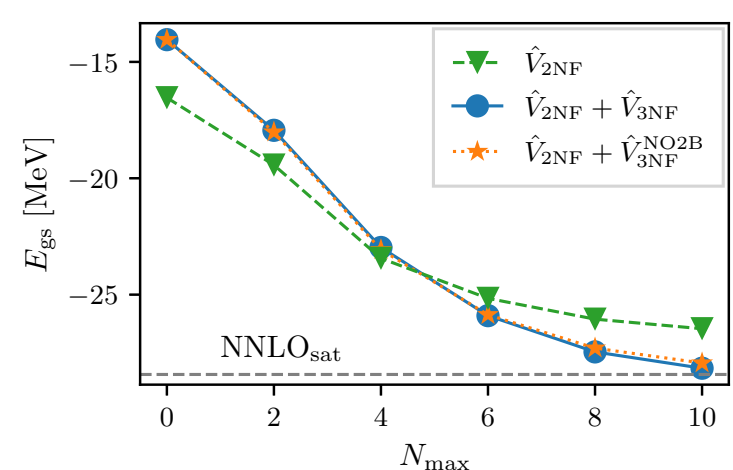

FIG. 3. The ground-state energy of ${ }^{4} \mathrm{He}$ computed with the $\mathrm{N} 2 \mathrm{LO}_{\text {sat }}$ interaction for fixed basis frequency $\hbar \Omega=20 \mathrm{MeV}$. The result with only the $2 \mathrm{NF}$ interaction (green, dashed line) is compared with the full Hamiltonian including 3NFs (blue, solid line) and with the NO2B-approximated one (orange, dotted line), corresponding to Eqs. (1) and (20), respectively. The dashed horizontal line indicates the converged $\mathrm{N} 2 \mathrm{LO}_{\text {sat }}$ ground-state energy $E_{\mathrm{gs}}=$ $-28.43 \mathrm{MeV}$ [32].

projection term acting on the $\mathrm{CM}$ coordinates. Instead, we will employ the metrics presented in Sec. III A as diagnostic tools.

\section{A. Ground-state energy of ${ }^{4} \mathrm{He}$}

We first compute the ground-state energy of ${ }^{4} \mathrm{He}$ at the fixed basis frequency $\hbar \Omega=20 \mathrm{MeV}$, which is close to the position of the variational minimum for this system with the $\mathrm{N}_{2} \mathrm{LO}_{\text {sat }}$ interaction. Results are shown in Fig. 3 as a function of increasing NCSM truncation $N_{\max }$ and compared to the converged result $E_{\mathrm{gs}}=-28.43 \mathrm{MeV}$ [32].

At this basis frequency, we find that the NO2B approximation captures the $N_{\max }$ behavior of the results obtained with the full Hamiltonian to within $1 \%$. This means that the approximation error in the total binding energy is smaller than $250 \mathrm{keV}$. We can also observe the importance of the $3 \mathrm{NF}$ since a full removal of this part of the Hamiltonian (green dashed line in Fig. 3) leads to approximately $2 \mathrm{MeV}$ underbinding.

However, the magnitude of the NO2B-approximation error turns out to be highly sensitive to the choice of basis frequency. This finding is highlighted in Fig. 4 where the binding energy per nucleon is computed for $\hbar \Omega \in$ $\{8,12, \ldots, 32,36\} \mathrm{MeV}$. The solid lines in the upper panel correspond to $E_{\mathrm{gs}} / A$ computed with the full Hamiltonian, while the dashed ones correspond to the NO2B-approximated $3 \mathrm{NF}$. The difference between these two results is shown in the lower panel as a function of the basis frequency.

There seems to be an optimal frequency $\hbar \Omega \approx 20 \mathrm{MeV}$ for which the approximation error is very small as we transition from under- to overbinding with the NO2B truncation. For higher frequencies there is an increasing difference between the NO2B and the full-3NF results. Note also that the NO2B truncation at $N_{\max }=0$ is identical to the full Hamiltonian as the single reference state is the only basis state.

The main hypothesis of this paper is that the explicitly broken translational symmetry of the NO2B Hamiltonian can
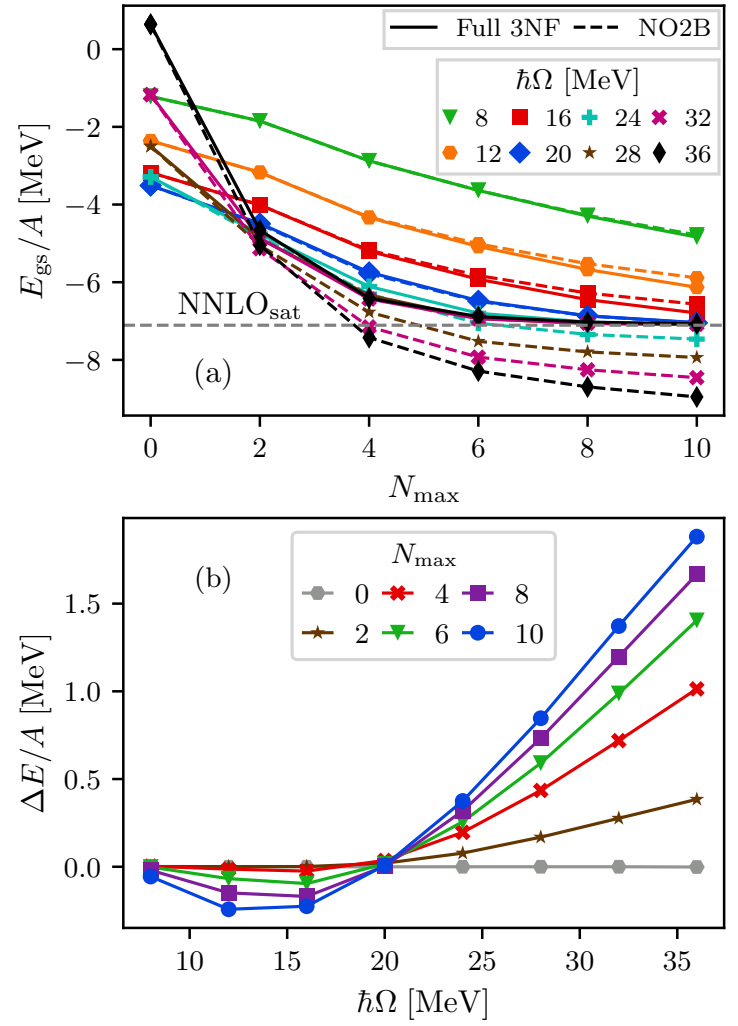

FIG. 4. (a) The ground-state energy per nucleon of ${ }^{4} \mathrm{He}$ computed with the $\mathrm{N} 2 \mathrm{LO}_{\text {sat }}$ interaction for different $\hbar \Omega$. The solid lines show results with full inclusion of the $3 \mathrm{NF}$, while the dashed lines correspond to NO2B-approximated 3NF. Although the convergence rate is $\hbar \Omega$ dependent, the full $3 \mathrm{NF}$ results converge towards the exact result (horizontal dashed line) while the results with the NO2B approximation do not. (b) The NO2B-approximation error $\Delta E \equiv$ $E_{\mathrm{gs}}^{3 \mathrm{NF}}-E_{\mathrm{gs}}^{\mathrm{NO} 2 \mathrm{~B}}$ per nucleon where $E_{\mathrm{gs}}^{3 \mathrm{NF}}$ and $E_{\mathrm{gs}}^{\mathrm{NO} 2 \mathrm{~B}}$ are the groundstate eigenenergies of Eqs. (1) and (20), respectively.

become the origin of a strong $\hbar \Omega$ dependence of the approximation error. Consequently, the NCSM eigenstates might not necessarily separate into a product of $\mathrm{CM}$ and intrinsic states.

To test this hypothesis we evaluate the $\mathrm{CM}$ metrics, $\xi_{\mathrm{CM}}$ and $N_{\mathrm{CM}}$-defined in Sec. III A-and the corresponding CM oscillator-state frequencies $\omega_{\xi}$ and $\omega_{N}$. These results are shown in Fig. 5. We observe that $\hbar \omega_{\xi} \gg \hbar \Omega$ for large basis frequencies. However, the $\xi_{\mathrm{CM}}$ metric clearly indicates that there is no CM separation in this scenario so the value of $\omega_{\xi}$ does not really have any significance.

In contrast, for small basis frequencies we have a clear factorization of the eigenstate, as indicated by both metrics, and we also find that the extracted frequencies are very similar and very close to the basis frequency. There is a transition region around $\hbar \Omega \approx 20 \mathrm{MeV}$ where the metrics indicate $\mathrm{CM}$ separation at a frequency that is slightly larger than the basis one.

The finding that $\mathrm{CM}$ mixing is less of a concern for small basis frequencies also indicates that the NO2B-approximation error of $\lesssim 500 \mathrm{keV} / A$ in this region is due to the neglected, residual $3 \mathrm{NF}$. As shown in the lower panels of Fig. 5 we find that both $\xi_{\mathrm{CM}}$ and $N_{\mathrm{CM}}$ do become very small for basis 

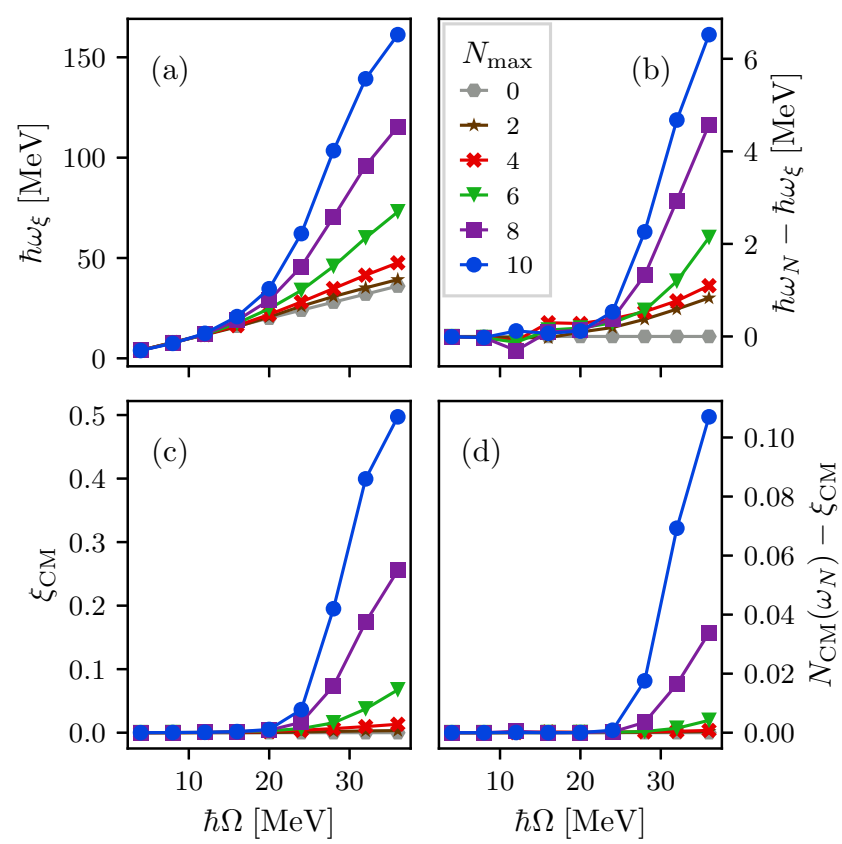

FIG. 5. CM analysis for ${ }^{4} \mathrm{He}$ eigenstates obtained with the NO2B Hamiltonian. The frequency $\hbar \omega_{\xi}$ from Eq. (27) is shown in panel (a) and the difference $\hbar \omega_{N}-\hbar \omega_{\xi}$ is shown in panel (b). Note that the basis frequency $\hbar \Omega$ is equal to both $\hbar \omega_{N}$ and $\hbar \omega_{\xi}$ at $N_{\max }=0$, as shown by the gray line with circle markers. The $\mathrm{CM}$ metric $\xi_{\mathrm{CM}}$ from Eq. (41) is shown in panel (c) and the difference $N_{\mathrm{CM}}\left(\omega_{N}\right)-\xi_{\mathrm{CM}}$ in panel (d)

frequencies below $\approx 20 \mathrm{MeV}$, indicating a separation between the $\mathrm{CM}$ and intrinsic parts of the ${ }^{4} \mathrm{He}$ ground state. However, as the basis frequency increases beyond $20 \mathrm{MeV}$ both measures increase drastically, suggesting that there is no longer any separation. The frequencies $\hbar \omega_{N}$ and $\hbar \omega_{\xi}$ start to differ visibly from the basis frequency already at $16 \mathrm{MeV}$, which is below the observed optimal frequency. This indicates that the $\mathrm{NO} 2 \mathrm{~B}$ approximation does affect the $\mathrm{CM}$ state, albeit very weakly.

While the approximation error at low basis frequencies can be attributed to neglected $3 \mathrm{NF}$ contributions, we note that this error is in general entangled with the effects of $\mathrm{CM}$ mixing such that the total approximation error cannot be written as a simple sum with two independent contributions. Furthermore, both these errors have a complicated, nonlinear dependence on the basis frequency via the reference state. The $3 \mathrm{NF}$ consists of both long- and short-distance contributions, which will be emphasized differently by reference states obtained with different basis frequencies. The observed optimal frequency $\hbar \Omega \approx 20 \mathrm{MeV}$ corresponds to a cancellation of residual 3NF and $\mathrm{CM}$ effects. We expect both these errors to increase with increasing $\hbar \Omega$.

\section{B. Ground-state energy of ${ }^{16} \mathrm{O}$}

We will now study the NO2B approximation when performing NCSM calculations of the ${ }^{16} \mathrm{O}$ nucleus. For this system we are limited to $N_{\max } \leqslant 8$ for Hamiltonians including only $2 \mathrm{NFs}$, and $N_{\max } \leqslant 6$ when using the Hamiltonian
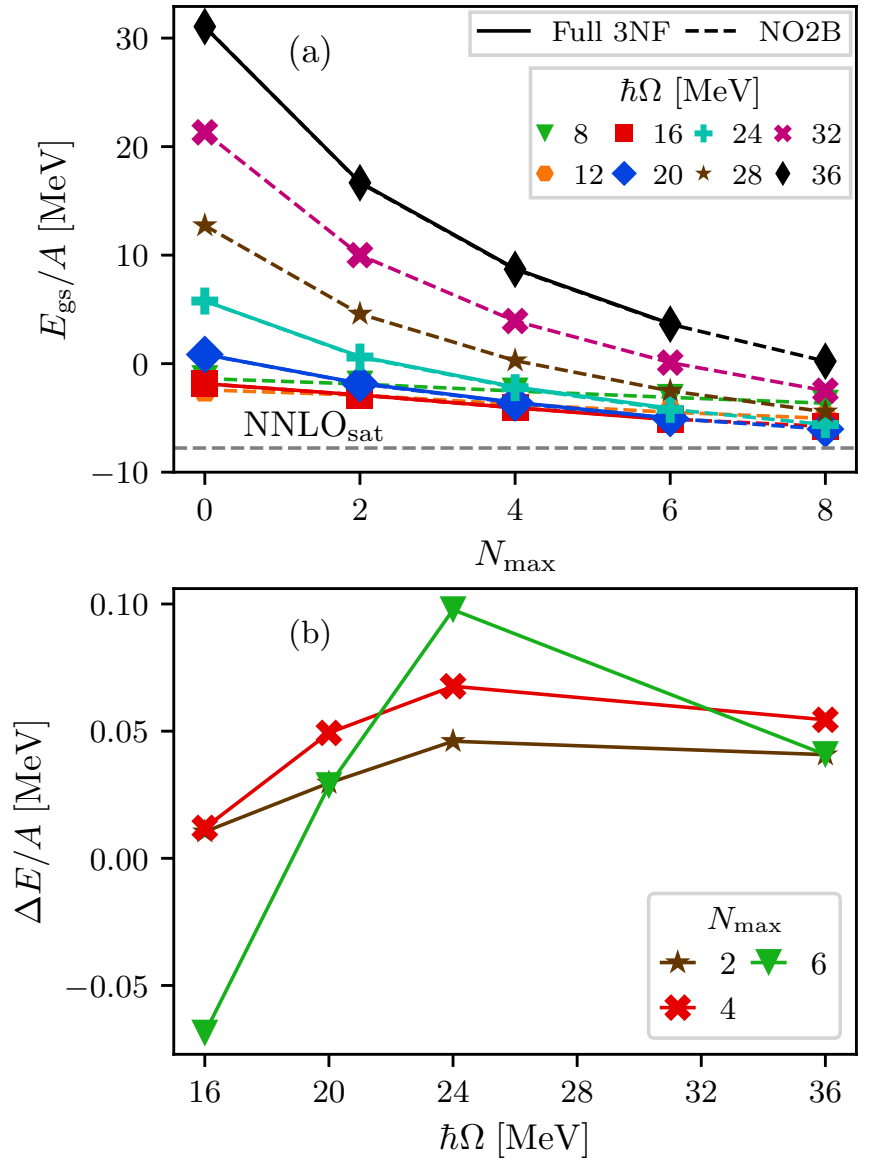

FIG. 6. (a) The ground-state energy per particle of ${ }^{16} \mathrm{O}$ computed with the $\mathrm{N} 2 \mathrm{LO}_{\text {sat }}$ interaction for different $\hbar \Omega$. The solid lines show results with full inclusion of the $3 \mathrm{NF}$ (only available for a subset of $\mathrm{HO}$ frequencies and up to $N_{\max }=6$ ), while the dashed lines correspond to NO2B-approximated $3 \mathrm{NF}$. The horizontal dashed line is the converged $\mathrm{N} 2 \mathrm{LO}_{\text {sat }}$ result computed with the $\mathrm{CC}$ method [32]. (b) The NO2B-approximation error $\Delta E \equiv E_{\mathrm{gs}}^{3 \mathrm{NF}}-E_{\mathrm{gs}}^{\mathrm{NO} 2 \mathrm{~B}}$ per particle where $E_{\mathrm{gs}}^{3 \mathrm{NF}}$ and $E_{\mathrm{gs}}^{\mathrm{NO} 2 \mathrm{~B}}$ are the ground-state eigenenergies of Eqs. (1) and (20), respectively.

with full 3NFs. Such differences in computational limits are the main reason for using the NO2B approximation in the first place. In this paper, NCSM computations with the full $3 \mathrm{NF}$ for ${ }^{16} \mathrm{O}$ are only performed at a few basis frequencies: $\hbar \Omega=16,20,24,36 \mathrm{MeV}$.

In addition, it is well known that $\mathrm{CM}$ effects are suppressed in heavier systems since the excitation of CM motion is energetically costly. Accordingly, in Fig. 6 we find that the NO2B approximation captures the $N_{\max }$ dependence of the groundstate energy results rather well for a wide frequency range. Note, however, that we are relatively far from convergence at $N_{\max }=8$ when using large basis frequencies. For comparison, we also show the converged result from $\mathrm{CC}$ calculations $E_{\mathrm{gs}} / A=-7.78 \mathrm{MeV}$ [32]

The lower panel of Fig. 6 shows that the NO2Bapproximation error is on the order of $\lesssim 100 \mathrm{keV} / A$, corresponding to $\approx 1.5 \mathrm{MeV}$ in the total binding energy (just over $1 \%)$. 

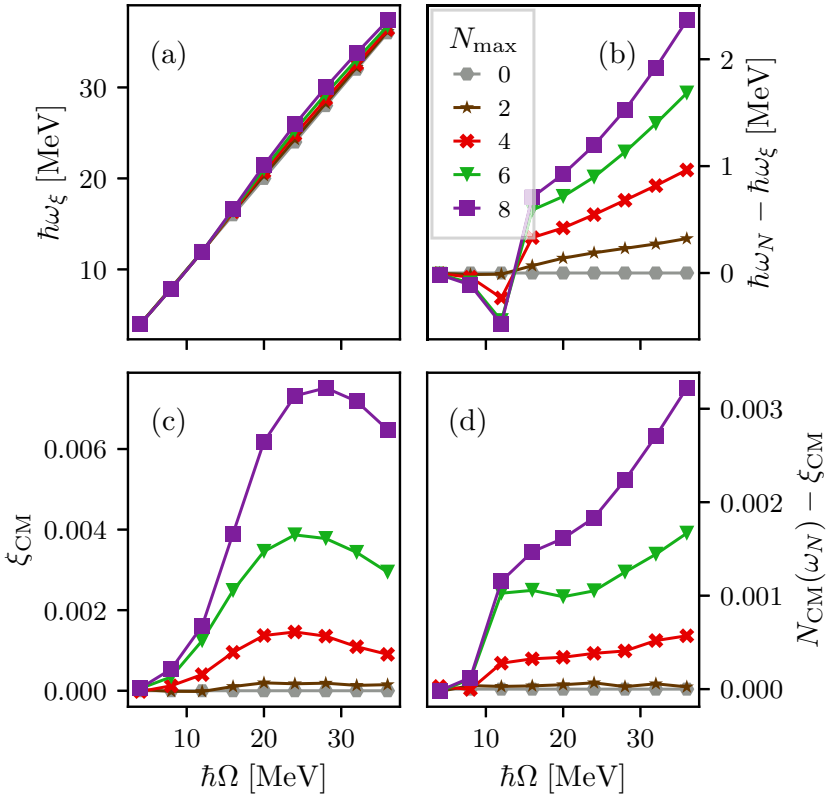

FIG. 7. $\mathrm{CM}$ analysis for ${ }^{16} \mathrm{O}$ eigenstates obtained with the NO2B Hamiltonian. The frequency $\hbar \omega_{\xi}$ from Eq. (27) is shown in panel (a) and the difference $\hbar \omega_{N}-\hbar \omega_{\xi}$ is shown in panel (b). Note that the basis frequency $\hbar \Omega$ is equal to both $\hbar \omega_{N}$ and $\hbar \omega_{\xi}$ at $N_{\max }=0$, as shown by the gray line with circle markers. The CM metric $\xi_{\mathrm{CM}}$ from Eq. (41) is shown in panel (c) and the difference $N_{\mathrm{CM}}\left(\omega_{N}\right)-\xi_{\mathrm{CM}}$ in panel (d).

The evaluated CM metrics are shown in Fig. 7, confirming the satisfactory factorization of the eigenstate. In fact, both $N_{\mathrm{CM}}$ and $\xi_{\mathrm{CM}}$ are orders of magnitude smaller for ${ }^{16} \mathrm{O}$ compared to ${ }^{4} \mathrm{He}$. Moreover, the HO frequency of the CM state is very close to the one for the basis across the frequency range we explore.

The small magnitude of $\mathrm{CM}$ effects in ${ }^{16} \mathrm{O}$ indicates that the total approximation error is mainly due to the residual $3 \mathrm{NF}$ that is neglected in the NO2B approximation. It is quite likely that the magnitude of this error will increase slightly with increasing $N_{\max }$, similar to observations for ${ }^{4} \mathrm{He}$ in Fig. 4(b). Finally it seems from Fig. 6(b) that $\hbar \Omega \lesssim 20 \mathrm{MeV}$ again represents an optimal frequency, but it would have been interesting to also have $N_{\max }=8$ results for ${ }^{16} \mathrm{O}$.

\section{Point proton radii of ${ }^{4} \mathrm{He}$ and ${ }^{16} \mathrm{O}$}

As a final set of results we also analyze the NO2Bapproximation error in the point-proton radii of ${ }^{4} \mathrm{He}$ and ${ }^{16} \mathrm{O}$ (see Figs. 8 and 9, respectively). For ${ }^{4} \mathrm{He}$ we find a rather large approximation error and-unlike the results for ground-state energies- there does not seem to exist an optimal frequency where the error is at a minimum.

For ${ }^{16} \mathrm{O}$ we find a good agreement between results with NO2B approximated and the full Hamiltonian, even though the point-proton radius shows a much slower convergence rate for very high basis frequencies. The approximation error is $\lesssim 0.01 \mathrm{fm}$. When comparing the radius predictions for ${ }^{4} \mathrm{He}$ and ${ }^{16} \mathrm{O}$ we again find that the $\mathrm{NO} 2 \mathrm{~B}$-approximation error and ensuing $\mathrm{CM}$ contamination decrease with increasing mass
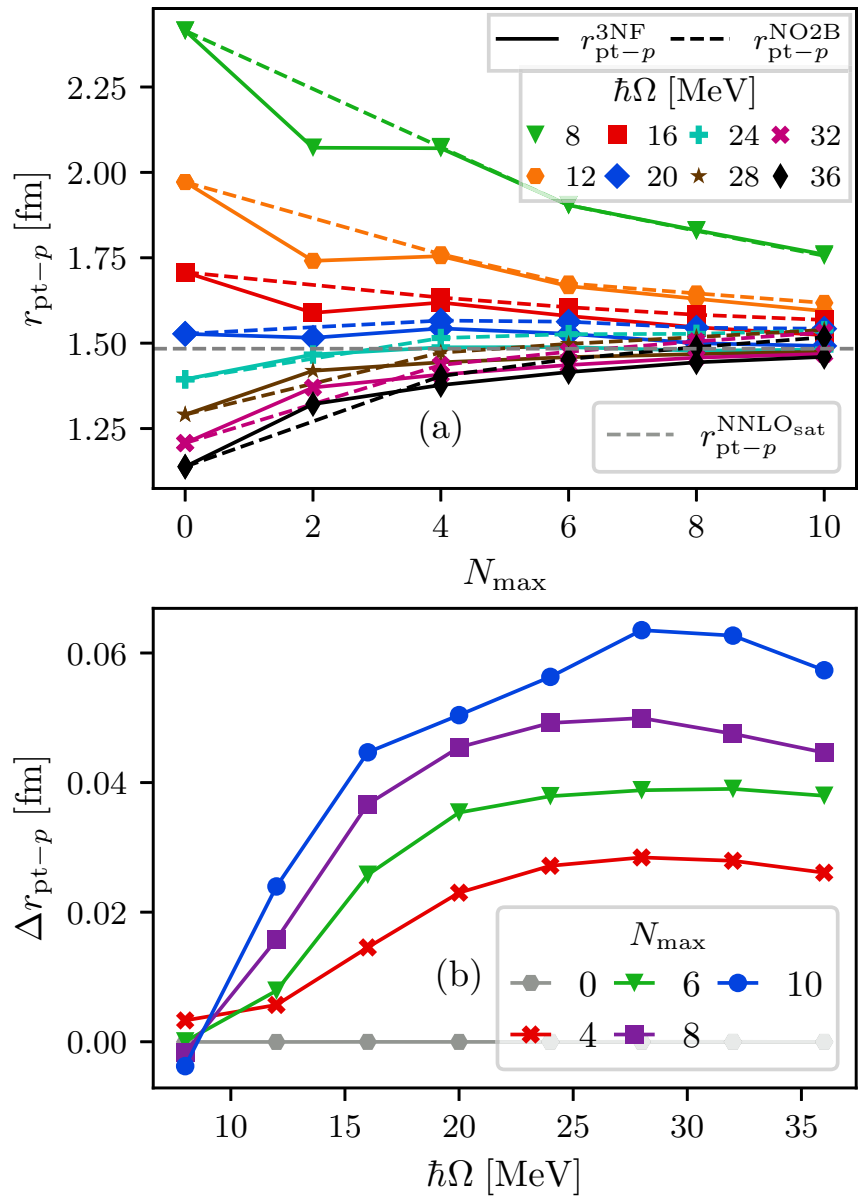

FIG. 8. The point-proton radius of ${ }^{4} \mathrm{He}$ computed with either full or NO2B-approximated 3NF. The large difference between low and high frequencies is due to slower convergence of NCSM calculations at low frequencies.

number, as expected. It is also interesting to note that the maximal NO2B-approximation error for the total energy per particle and point-proton radius are both reduced by roughly a factor of 10 when going from $A=4$ to 16 , at least for the range of oscillator frequencies that we explore here.

\section{DISCUSSION}

There is a dilemma between the need to include 3NFs in nuclear calculations to achieve increased physical accuracy and precision, and the significant increase in computational complexity caused solely by the inclusion of 3NFs in $a b$ initio methods. In this paper we have studied the SR-NO2B approximation of $3 \mathrm{NFs}$, that aims to reduce the computational complexity to that of $2 \mathrm{NFs}$ while still capturing the most important effects of the $3 \mathrm{NF}$ physics. Our study is performed in the framework of the NCSM method.

The SR-NO2B approximation utilizes Wick's theorem to expand the 3NF potential in a sum of constant, one-, two-, and three-body operators that are normal ordered relative to a nonvacuum reference state $\left|\Psi_{\text {ref }}\right\rangle$, taken to be a single SD. In this paper the reference state is constructed in the $\mathrm{HO}$ basis and we explore the sensitivity of computed observables to 

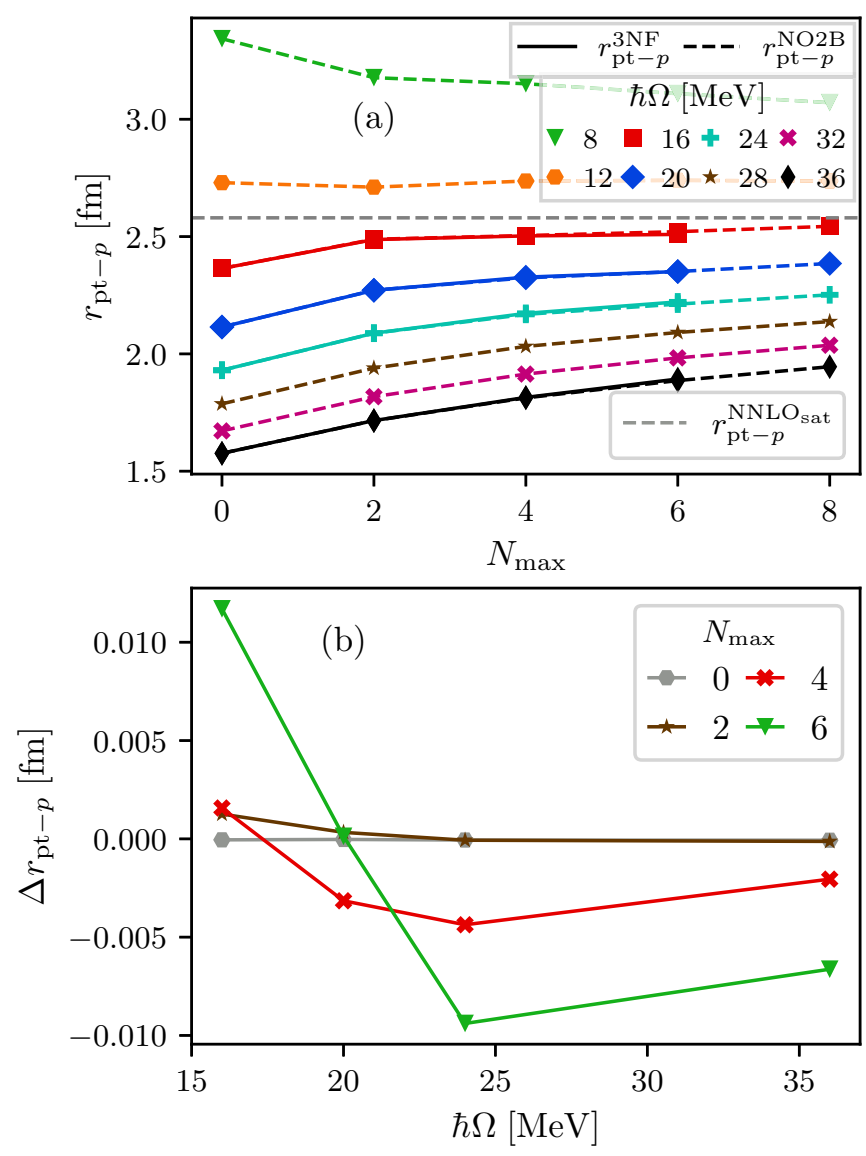

FIG. 9. The point-proton radius of ${ }^{16} \mathrm{O}$ computed with either full or NO2B-approximated 3NF. The large difference between low and high frequencies is due to slower convergence of NCSM calculations at low frequencies.

the choice of the basis frequency. If the reference state is a good approximation to the ground state of the nucleus, then the normal-ordered three-body term can be discarded as it is legitimate to assume that it will have a negligible contribution to the ground-state energy.

A problem with the SR-NO2B approximation is that it breaks the translational symmetry of the underlying Hamiltonian. In this paper we have focused on the consequences of this symmetry breaking by introducing CM metrics and studying the $\mathrm{NO} 2 \mathrm{~B}$-approximation error for energies and radii of ${ }^{4} \mathrm{He}$ and ${ }^{16} \mathrm{O}$.

The main findings and conclusions of this study are as follows.

(1) Translational invariance is explicitly broken in the $N O 2 B$ approximation. The truncation of the normal-ordered Hamiltonian operator introduces a $\mathrm{CM}$ dependence of the reference state, which can lead to $\mathrm{CM}$ mixing even if a totalenergy truncated NCSM basis is used.

(2) Metrics are important for assessing the CM mixing in eigenstates obtained with the SR-NO2B approximation. We have found that the previously introduced $\xi_{\mathrm{CM}}$ and $N_{\mathrm{CM}}$ metrics are useful for this purpose.

(3) The comparison of different CM metrics can reveal more information about the details of the CM factorization. Zero metrics, $\xi_{\mathrm{CM}}=N_{\mathrm{CM}}\left(\omega_{\xi}\right)=0$, imply proper CM factorization with the CM part in its ground state. However, nonzero metrics do not help us determine whether we have a mixed state or a linear superposition.

(4) The ability of the NO2B approximation to describe the ${ }^{4} \mathrm{He}$ ground-state energy depends strongly on the NCSM basis frequency $\hbar \Omega$. The NO2B-approximation error is the smallest for $\hbar \Omega=20 \mathrm{MeV}$, but it increases significantly for larger basis frequencies. A very weak dependence is observed for smaller frequencies. Both CM metrics indicate negligible CM mixing at small frequencies, and strongly increasing mixing at large ones.

(5) The CM problem is much less significant for the ground state of ${ }^{16} \mathrm{O}$. For this system the differences between the $\mathrm{NO} 2 \mathrm{~B}$-approximated ground-state energies and the full-3NF ones do not exhibit any significant basis-frequency dependency. Furthermore, there seems to be no significant CM mixing, since both $\xi_{\mathrm{CM}}$ and $N_{\mathrm{CM}}$ are small.

(6) We recommend further investigations of the CM problem in the $\mathrm{NO} 2 \mathrm{~B}$ approximation also when using other basis functions. In this paper we have focused on the SR-NO2B approximation with a $\mathrm{HO}$ basis. However, reference states constructed from other single-particle bases might yield better results. In particular the Hartree-Fock and the natural orbit bases are being used in some many-body solvers and results could be analyzed in a similar fashion as in this paper.

(7) A general analysis of CM factorization can be performed with the squared $C M$ density matrix. The metrics, $\xi_{\mathrm{CM}}$ and $N_{\mathrm{CM}}$, used in this paper can only detect a CM factorization if the CM wave function is a Gaussian. A more general approach might be to consider the trace of the squared CM density matrix. We will only have a pure CM quantum state if $\operatorname{tr}\left\{\rho_{\mathrm{CM}}^{2}\right\}=1$; in every other case $\operatorname{tr}\left\{\rho_{\mathrm{CM}}^{2}\right\}<1$. However, in practice this analysis method requires the trace to be computed in a sufficiently large basis.

(8) Expectation values of other observables than groundstate energies can be strongly affected by the use of the $N O 2 B$ approximation. Expectation values are computed with respect to the eigenstates, and might therefore exhibit a stronger CMmixing effect. While we did compute the approximation error for point-proton radii-and found that it was particularly large for ${ }^{4} \mathrm{He}$ - the general effects of the NO2B approximation on other observables were not fully analyzed in this paper.

\section{ACKNOWLEDGMENTS}

We thank S. R. Stroberg for useful discussions and suggestions. We thank P. Navrátil for useful discussions and for support in the use of the NCSD code. This work was supported by the Swedish Research Council (Grant No. 201704234) and the European Research Council (ERC) under the European Unions Horizon 2020 Research and Innovation Programme (Grant No. 758027). The computations were enabled by resources provided by the Swedish National Infrastructure for Computing at Chalmers Centre for Computational Science and Engineering, the National Supercomputer Centre, partially funded by the Swedish Research Council. G.R.J. acknowledges support by the U.S. Department of Energy under Grant No. desc0018223 (NUCLEI SciDAC-4 


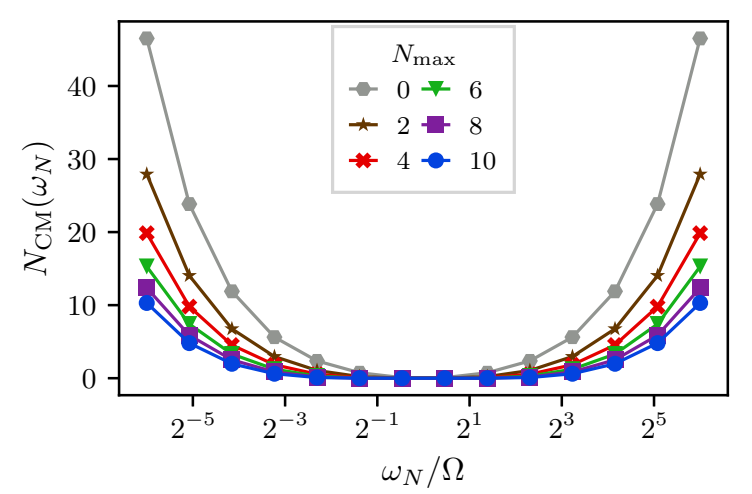

FIG. 10. The smallest eigenvalue $N_{\mathrm{CM}}\left(\omega_{N}\right)$, of the CM HO number operator $\hat{N}_{\mathrm{CM}}\left(\omega_{N}\right)$, see Eq. (23), computed in NCSM-bases with different basis frequencies $\Omega$.

collaboration). This research used resources of the Oak Ridge Leadership Computing Facility located at Oak Ridge National Laboratory, which is supported by the Office of Science of the U.S. Department of Energy under Contract No. DE-AC05-00OR22725. This manuscript has been authored by UT-Battelle, LLC under Contract No. DE-AC05-00OR22725 with the U.S. Department of Energy. The United States Government retains and the publisher, by accepting the article for publication, acknowledges that the United States Government retains a non-exclusive, paid-up, irrevocable, world-wide license to publish or reproduce the published form of this manuscript, or allow others to do so, for United States Government purposes. The Department of Energy will provide public access to these results of federally sponsored research in accordance with the DOE Public Access Plan [40].

\section{APPENDIX: REPRESENTATIONS IN A TRUNCATED BASIS}

It is not possible to fully represent a $\mathrm{HO}$ ground state with a frequency $\omega_{N}$ in a truncated HO basis with frequency $\Omega \neq$ $\omega_{N}$. Therefore, it is possible for the metrics $N_{\mathrm{CM}}\left(\omega_{N}\right)$ and $\xi_{\mathrm{CM}}$ to be nonzero even if the eigenstate is factorized:

$$
\left|\Psi_{\mathrm{GS}}^{\mathrm{NO} 2 \mathrm{~B}}\right\rangle_{\mathrm{NCSM}}=\left|\Psi_{\mathrm{GS}}\right\rangle_{\text {int }} \otimes\left|\Psi_{\mathrm{GS}}\right\rangle_{\mathrm{CM}}
$$

where $\left|\Psi_{\mathrm{GS}}\right\rangle_{\mathrm{CM}}$ is a $\mathrm{HO}$ ground state with frequency $\omega_{N}$. Here we will study eigenstates of the HO CM Hamiltonian (22). In particular, $N_{\mathrm{CM}}\left(\omega_{N}\right)$-which is the smallest eigenvalue to $\hat{N}_{\mathrm{CM}}\left(\omega_{N}\right)$ in the current, truncated NCSM basis-is shown in
Fig. 10 as a function of $\omega_{N} / \Omega$. Note that the horizontal axis is logarithmic. It is obvious that this metric is not necessarily zero even if we have a factorized product state.

We also observe in Fig. 10 that $N_{\mathrm{CM}}\left(\omega_{N}\right)$ is invariant under the transformation $\frac{\omega_{N}}{\Omega} \rightarrow \frac{\Omega}{\omega_{N}}$. Here we will demonstrate this algebraically. The analytical expression for the matrix elements $\hat{H}_{\mathrm{CM}}\left(\omega_{N}\right)$ in the CM-coordinate HO basis $|\mathcal{N}, \mathcal{L}\rangle$ with frequency $\Omega$ is

$$
\begin{aligned}
\left\langle\mathcal{N}^{\prime},\right. & \left.\mathcal{L}^{\prime}\left|\hat{H}_{\mathrm{CM}}\left(\omega_{N}\right)\right| \mathcal{N}, \mathcal{L}\right\rangle \\
= & \left\{\left(1+\frac{\omega_{N}{ }^{2}}{\Omega^{2}}\right) \frac{\hbar \Omega}{2}(2 \mathcal{N}+\mathcal{L}+3 / 2) \delta_{\mathcal{N}^{\prime}, \mathcal{N}}\right. \\
& -\frac{3}{2} \hbar \omega_{N} \delta_{\mathcal{N}^{\prime} \mathcal{N}}+\left(1-\frac{\omega_{N}^{2}}{\Omega^{2}}\right) \frac{\hbar \Omega}{2} \\
& \times\left[\sqrt{\mathcal{N}(\mathcal{N}+\mathcal{L}+1 / 2)} \delta_{\mathcal{N}^{\prime}+1, \mathcal{N}}\right. \\
& \left.\left.+\sqrt{\mathcal{N}^{\prime}\left(\mathcal{N}^{\prime}+\mathcal{L}+1 / 2\right)} \delta_{\mathcal{N}^{\prime}, \mathcal{N}+1}\right]\right\} \delta_{\mathcal{L}^{\prime}, \mathcal{L}}
\end{aligned}
$$

Therefore the matrix elements of $\hat{N}_{\mathrm{CM}}\left(\omega_{N}\right)$ can be written

$$
\begin{aligned}
& \left\langle\mathcal{N}^{\prime}, \mathcal{L}^{\prime}\left|\hat{N}_{\mathrm{CM}}\left(\omega_{N}\right)\right| \mathcal{N}, \mathcal{L}\right\rangle= \\
& =\frac{1}{2}\left\{\left(\frac{\Omega}{\omega_{N}}+\frac{\omega_{N}}{\Omega}\right)(2 \mathcal{N}+\mathcal{L}+3 / 2) \delta_{\mathcal{N}^{\prime}, \mathcal{N}}\right. \\
& \quad-\frac{3}{2} \delta_{\mathcal{N}^{\prime} \mathcal{N}}+\left(\frac{\Omega}{\omega_{N}}-\frac{\omega_{N}}{\Omega}\right) \\
& \quad \times\left[\sqrt{\mathcal{N}(\mathcal{N}+\mathcal{L}+1 / 2)} \delta_{\mathcal{N}^{\prime}+1, \mathcal{N}}\right. \\
& \left.\left.\quad+\sqrt{\mathcal{N}^{\prime}\left(\mathcal{N}^{\prime}+\mathcal{L}+1 / 2\right)} \delta_{\mathcal{N}^{\prime}, \mathcal{N}+1}\right]\right\} \delta_{\mathcal{L}^{\prime}, \mathcal{L}} .
\end{aligned}
$$

The diagonal is invariant under the transformation $\frac{\omega_{N}}{\Omega} \rightarrow \frac{\Omega}{\omega_{N}}$, but the off-diagonal terms change sign. However, since the matrix is symmetric and tridiagonal, the off-diagonal terms will be squared in the characteristic equation, eliminating the sign change. Thus the characteristic equation is invariant for $\frac{\omega_{N}}{\Omega} \rightarrow \frac{\Omega}{\omega_{N}}$. It follows then that the eigenvalues must be invariant too. Since $N_{\mathrm{CM}}\left(\omega_{N}\right)$ is the lowest eigenvalue this demonstrates that it also must be invariant under $\frac{\omega_{N}}{\Omega} \rightarrow \frac{\Omega}{\omega_{N}}$ in accordance with Fig. 10.

If $N_{\mathrm{CM}}\left(\omega_{N}\right) \approx N_{\mathrm{CM}}\left(\omega_{N}\right)$ then $\left|\Psi_{\mathrm{GS}}^{\mathrm{NO} 2 \mathrm{~B}}\right\rangle_{\mathrm{NCSM}}$ is separated in a HO-CM ground state and some intrinsic state. If, on the other hand, $N_{\mathrm{CM}}\left(\omega_{N}\right) \gg N_{\mathrm{CM}}\left(\omega_{N}\right)$ then the CM state is not a $\mathrm{HO}$ ground state.
[1] H.-W. Hammer, A. Nogga, and A. Schwenk, Rev. Mod. Phys. 85, 197 (2013).

[2] J. Fujita and H. Miyazawa, Prog. Theor. Phys. 17, 360 (1957).

[3] E. Epelbaum, Prog. Part. Nucl. Phys. 57, 654 (2006).

[4] E. Epelbaum, H.-W. Hammer, and Ulf-G. Meissner, Rev. Mod. Phys. 81, 1773 (2009).

[5] R. Machleidt and D. R. Entem, Phys. Rep. 503, 1 (2011).

[6] H. W. Hammer, S. König, and U. van Kolck, Rev. Mod. Phys. 92, 025004 (2020).
[7] N. Kalantar-Nayestanaki, E. Epelbaum, J. G. Messchendorp, and A. Nogga, Rep. Prog. Phys. 75, 016301 (2012).

[8] J. A. Tjon, Phys. Lett. B 56, 217 (1975).

[9] S. Wesolowski, I. Svensson, A. Ekström, C. Forssén, R. J. Furnstahl, J. A. Melendez, and D. R. Phillips, arXiv:2104.04441 [nucl-th].

[10] P. Navrátil and W. E. Ormand, Phys. Rev. C 68, 034305 (2003).

[11] B. R. Barrett, P. Navratil, and J. P. Vary, Prog. Part. Nucl. Phys. 69, 131 (2013). 
[12] J. Carlson, S. Gandolfi, F. Pederiva, S. C. Pieper, R. Schiavilla, K. E. Schmidt, and R. B. Wiringa, Rev. Mod. Phys. 87, 1067 (2015).

[13] J. P. Vary, P. Maris, E. Ng, C. Yang, and M. Sosonkina, J. Phys.: Conf. Series 180, 012083 (2009).

[14] G. Hagen, T. Papenbrock, D. J. Dean, A. Schwenk, A. Nogga, M. Wloch, and P. Piecuch, Phys. Rev. C 76, 034302 (2007).

[15] R. Roth, S. Binder, K. Vobig, A. Calci, J. Langhammer, and P. Navratil, Phys. Rev. Lett. 109, 052501 (2012).

[16] G. C. Wick, Phys. Rev. 80, 268 (1950).

[17] S. Binder, J. Langhammer, A. Calci, and R. Roth, Phys. Lett. B 736, 119 (2014).

[18] G. Hagen, T. Papenbrock, M. Hjorth-Jensen, and D. J. Dean, Rep. Prog. Phys. 77, 096302 (2014).

[19] G. R. Jansen, J. Engel, G. Hagen, P. Navratil, and A. Signoracci, Phys. Rev. Lett. 113, 142502 (2014).

[20] H. Hergert, S. K. Bogner, T. D. Morris, A. Schwenk, and K. Tsukiyama, Phys. Rep. 621, 165 (2016).

[21] A. Cipollone, C. Barbieri, and P. Navrátil, Phys. Rev. Lett. 111, 062501 (2013).

[22] E. Gebrerufael, A. Calci, and R. Roth, Phys. Rev. C 93, 031301(R) (2016).

[23] S. Binder, P. Piecuch, A. Calci, J. Langhammer, P. Navrátil, and R. Roth, Phys. Rev. C 88, 054319 (2013).

[24] D. Gloeckner and R. Lawson, Phys. Lett. B 53, 313 (1974).

[25] G. Hagen, T. Papenbrock, and D. J. Dean, Phys. Rev. Lett. 103, 062503 (2009).

[26] R. Roth, J. R. Gour, and P. Piecuch, Phys. Lett. B 679, 334 (2009).
[27] N. M. Parzuchowski, S. R. Stroberg, P. Navrátil, H. Hergert, and S. K. Bogner, Phys. Rev. C 96, 034324 (2017).

[28] A. Tichai, J. Müller, K. Vobig, and R. Roth, Phys. Rev. C 99, 034321 (2019).

[29] J. Hoppe, A. Tichai, M. Heinz, K. Hebeler, and A. Schwenk, Phys. Rev. C 103, 014321 (2021).

[30] J. Ripoche, A. Tichai, and T. Duguet, Eur. Phys. J. A 56, 40 (2020).

[31] M. Frosini, T. Duguet, B. Bally, Y. Beaujeault-Taudière, J. P. Ebran, and V. Somà, Eur. Phys. J. A 57, 151 (2021).

[32] A. Ekström, G. R. Jansen, K. A. Wendt, G. Hagen, T. Papenbrock, B. D. Carlsson, C. Forssén, M. Hjorth-Jensen, P. Navrátil, and W. Nazarewicz, Phys. Rev. C 91, 051301(R) (2015).

[33] W. H. Dickhoff and D. Van Neck, Many-Body Theory Exposed!: Propagator Description of Quantum Mechanics in Many-Body Systems, 2nd ed. (World Scientific, Singapore, 2008).

[34] D. Lee, Prog. Part. Nucl. Phys. 63, 117 (2009).

[35] P. Navrátil, G. P. Kamuntavičius, and B. R. Barrett, Phys. Rev. C 61, 044001 (2000).

[36] A. Ekström, G. Baardsen, C. Forssén, G. Hagen, M. HjorthJensen, G. R. Jansen, R. Machleidt, W. Nazarewicz, T. Papenbrock, J. Sarich, and S. M. Wild, Phys. Rev. Lett. 110, 192502 (2013).

[37] P. Navrátil and E. Caurier, Phys. Rev. C 69, 014311 (2004).

[38] C. Forssén, B. D. Carlsson, H. T. Johansson, D. Sääf, A. Bansal, G. Hagen, and T. Papenbrock, Phys. Rev. C 97, 034328 (2018).

[39] P. Navrátil, computer code No-Core Shell Model Slater Determinant (NCSD), 1995 (unpublished).

[40] http://energy.gov/downloads/doe-public-access-plan. 